\title{
Relationship Among Homocysteine, Inflammation and Cognitive Impairment in Patients with Acute Ischemic Stroke and Transient Ischemic Attack
}

\author{
Lingyun Cui (D) \\ Ping Lu' \\ Shiyu $\mathrm{Li}^{\prime}$ \\ YueSong $\mathrm{Pan}^{2}$ \\ Mengxing Wang ${ }^{2}$ \\ Zixiao $\mathrm{Li}^{1,2}$ \\ Xiaoling Liao ${ }^{1,2}$ \\ Yongjun Wang ${ }^{1,2}$ \\ 'Department of Neurology, Beijing \\ Tiantan Hospital, Capital Medical \\ University, Beijing, People's Republic of \\ China; ${ }^{2}$ China National Clinical Research \\ Center for Neurological Diseases, \\ Beijing, People's Republic of China
}

Correspondence: Yongjun Wang Department of Neurology, Beijing Tiantan Hospital, Capital Medical University, I I9\#Nan Si Huan Xi Lu, Beijing, 100070, People's Republic of China Tel +86 I0-5997835 I

Email yongjunwang@ncrend.org.cn
Purpose: To investigate the associations among homocysteine (Hcy), inflammation and cognitive impairment in patients with acute ischemic stroke (AIS) and transient ischemic attack (TIA).

Patients and Methods: Patients included were enrolled from a subgroup of China National Stroke Registry-III (CNSR-III). We used a Chinese version of Montreal Cognitive Assessment (MoCA) to screen for cognitive impairment. We used high-sensitivity C-reactive protein (hsCRP) level to reflect the inflammatory status, which was assessed at baseline together with Hcy concentration. The primary outcome was the incidence of post-stroke cognitive impairment (PSCI) at 3 months after AIS and TIA. Multivariable logistic regression analysis was used to evaluate the correlation between Hcy and hsCRP, and their effects on cognition.

Results: We enrolled 1466 patients with a median age of 62 (54-70) years old, including 895 (61.05\%) patients with elevated Hcy levels, 466 (31.79\%) with increased hsCRP concentrations, and $755(51.50 \%)$ with PSCI. In the group of patients with hyperhomocysteinemia (HHcy), higher hsCRP levels were related to cognitive impairment, whether or not adjusted for multiple potential confounders (crude OR: 1.71,95\% CI: 1.29-2.27, p < 0.01; adjusted OR: $1.42,95 \% \mathrm{CI}: 1.04-1.93, \mathrm{p}=0.03)$. No significant interactions for the impact on PSCI were observed in subgroups stratified by age, sex or Trial of Org 10172 in Acute Stroke Treatment (TOAST) classification (P interaction $>0.05$ for all).

Conclusion: High inflammatory levels increase the risk of cognitive impairment in HHcy patients after AIS and TIA.

Keywords: stroke, transient ischemic attack, inflammation, homocysteine, cognitive function

\section{Introduction}

In addition to its high lethality, stroke is also recognized as a vital contributor to disability and cognitive impairment. ${ }^{1,2}$ Among them, cognitive impairment significantly delays functional recovery and affects the life quality of patients, ${ }^{3-5}$ as well as increases healthcare costs. Therefore, early and accurate identification of risk factors associated with cognitive impairment to improve the prediction of PSCI is critical for optimizing the treatments.

Serum homocysteine, as a kind of sulfur-containing amino acids, is identified as a reactive vascular injury amino acid due to the strong oxidative effects on vascular endothelial cells and subsequent vascular lesions. ${ }^{6}$ Meanwhile, serum Hcy levels are affected by several factors, including age, sex (male), pregnancy, high methionine-rich protein diet, vitamin (such as folic acid, vitamin B6, and B12) deficiency, renal 
failure, ${ }^{7,8}$ genetic mutation including heterozygous/homozygous cystathionine- $\beta$ synthase (CBS) gene and MTHFR gene, medication (such as methotrexate and niacin) use, and so on, ${ }^{9,10}$ all of which can contribute to HHcy. As a risk factor for cardiovascular diseases, Hcy is also closely related to multiple neurological diseases such as stroke and cognitive decline. ${ }^{11,12}$ In recent years, emerging evidence has been discovered to support the link between homocysteine and PSCI, ${ }^{13,14}$ among which inflammatory pathway is regarded as one of the essential underlying mechanisms. As an acute-phase protein and the most sensitive biomarker of nonspecific inflammatory response, hsCRP is elevated in patients with cognitive impairment, ${ }^{15,16}$ and can independently predict PSCI, ${ }^{17}$ thus exhibiting the potential for evaluating cognitive disorders in clinics. ${ }^{18}$

To our knowledge, no individual study has analyzed the relationships among homocysteine, hsCRP and PSCI. In this study, we aim to investigate their relationship based on CNSR-III in patients with AIS and TIA.

\section{Patients and Methods}

\section{Study Design and Subjects}

We obtained the data from the Impairment of Cognition and Sleep (ICONS) subgroup of CNSR-III. ${ }^{19}$ The ICONS study is a multicenter (40 hospitals) prospective registry. It recruited AIS or TIA inpatients (within 7 days of onset) between August 15, 2015 and January 2018, with 1-year follow-up finished in March 2019 and 2625 patients were included. Patients who met the preplanned inclusion criteria were eligible: age over 18 years old; in-hospital AIS or TIA without any history of severe cognitive impairment prior to stroke or any comorbidity that could interfere with cognitive assessment. After excluding 825 individuals with missing information on Hcy,84 individuals with missing information on hsCRP at baseline and 250 individuals without MoCA assessments at 3-month follow-up, we finally included 1466 subjects for subsequent analysis (Figure 1).

Prior to data collection, we have obtained approval from the ethics committee of Beijing Tiantan Hospital, and signed informed consent from all included patients.

\section{Baseline Data}

Patients with AIS or TIA with complete data of baseline Hcy and hsCRP levels as well as cognitive evaluation at 3-month by trained neurologists were included. Other baseline characteristics including age, gender, body mass index (BMI) as well as past history were collected by a professional physician during the interview. We dichotomized the levels of Hcy and hsCRP as follows, normal

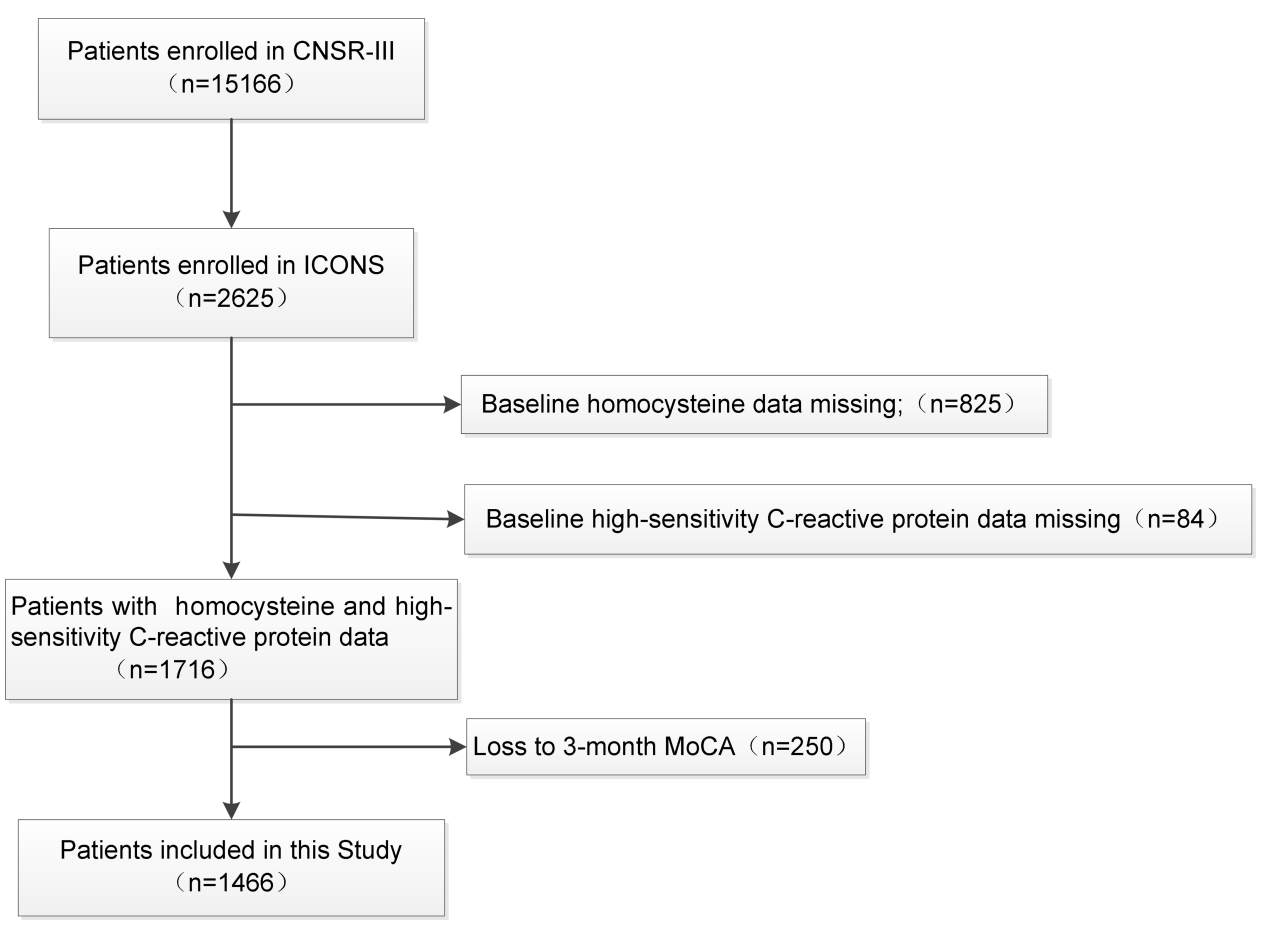

Figure I Patient Flowchart. 
Hcy $(<15 \mathrm{mmol} / \mathrm{L})$, HHcy $(\geq 15 \mathrm{mmol} / \mathrm{L}),{ }^{20}$ normal hsCRP $(<3 \mathrm{mg} / \mathrm{L})$, and high hsCRP $(\geq 3 \mathrm{mg} / \mathrm{L}){ }^{21}$

\section{Outcome Assessment}

The MoCA was employed to detect PSCI and assess global cognition. ${ }^{22}$ Specifically, a patient with a MoCA score below 26, or less than 12 years of education and a MOCA score below 25 was considered cognitively impaired. ${ }^{23}$ The primary outcome was the prevalence of PSCI at 3 months after the onset of AIS or TIA.

\section{Statistical Analysis}

The data analyses were conducted using SAS 9.4 (SAS Institute Inc, Cary, NC). Continuous variables were exhibited as medians with interquartile ranges and categorical variables as percentages. For comparisons, we used one-way analysis of variance or KruskalWallis test for the continuous variables and chi-square statistics for the categorical variables. We employed multivariable logistic regression analysis to evaluate the correlations among hsCRP, Hcy, and PSCI. Results were reported as odds ratios (ORs) with 95\% confidence interval $(95 \% \mathrm{CI}) . \mathrm{P}<0.05$ was considered statistically significant.

\section{Results}

\section{Demographic and Clinical Characteristics}

We selected 1466 eligible patients from the 2625 patients in the ICONS study with a median age of 62 years old. Four hundred and fourteen $(28.24 \%)$ of them were female. Compared with patients excluded, the included patients had higher rates of history of hyperlipidemia but lower BMI, as shown in Table 1.

Baseline characteristics of study patients are displayed in Table 2. Among participants included in our study, 466 (31.79\%) patients had higher hsCRP levels. Compared with the NCRP (normal high-sensitivity C-reactive protein) group, more patients in the HCRP (high high-sensitivity C-reactive protein) group had elevated Hcy levels. The median Hcy levels were $16.5 \mu \mathrm{mol} / \mathrm{L}$ (NCRP group) and $16.6 \mu \mathrm{mol} / \mathrm{L}$ (HCRP groups), respectively. Participants in the HCRP groups were older, more likely to have higher proportions of history of hypertension and diabetes, had higher systolic blood pressure at admission, furthermore, they had higher baseline white blood cell (WBC) counts and
Vitamin B12 levels. While, in the HHcy group, patients with increased hsCRP concentrations were older, had higher WBC counts, folate, and vitamin B12 levels, as well as higher baseline NIHSS. While in the NHcy group, patients with increased hsCRP concentrations were older, more likely to have higher proportions of history of hypertension and diabetes, had higher baseline WBC counts and NIHSS score.

\section{Clinical Outcomes}

Table 3 exhibits the comparison of outcomes between the groups. No significant differences of the PSCI were found between the NCRP and HCRP groups after adjusting for potential variables (crude OR: 1.54,95\% CI: $1.23-1.92, \mathrm{p}<0.001$; adjusted OR: $1.27,95 \% \mathrm{CI}$ : $0.99-1.62, p=0.06)$. In the HHcy group, patients with high hsCRP level were significantly likely to suffer from cognitive impairment, even if the confounding factors were controlled (crude OR: $1.71,95 \%$ CI: $1.29-2.27, \mathrm{p}<0.01$; adjusted OR: $1.42,95 \% \mathrm{CI}$ : 1.04-1.93, $\mathrm{p}=0.03)$. However, no similar correlation in the NHcy group was identified (crude OR: 1.30, 95\% CI: 0.91-1.87, $\mathrm{p}=0.15$; adjusted OR: $1.05,95 \%$ CI: $0.70-1.58, \mathrm{p}=0.80$ ). When comparing the four groups directly, only the high hsCRP and high homocysteine groups were related to cognitive impairment, but this association was attenuated after adjustment for potential confounders (crude OR: 1.48, 95\% CI: $1.09-$ $2.01, \mathrm{p}=0.01$; adjusted OR: $1.23,95 \%$ CI: $0.88-1.72$, $\mathrm{p}=0.23$ ). Furthermore, after classifying patients according to age, sex, and TOAST criteria, those who were older (adjusted OR: 1.45, 95\% CI: 1.02-2.05, $\mathrm{p}=$ 0.04), male (adjusted OR: 1.67, 95\% CI: 1.05-2.65, $\mathrm{p}=0.03$ ), or large-artery atherosclerosis (LAA) classification (adjusted OR: 1.96, 95\% CI: 1.05-3.66, $\mathrm{p}=$ 0.04 ) were more likely to have cognitive impairment if presenting high homocysteine and high hsCRP levels at the same time (Table 4). Of note, no significant interaction for the impact on PSCI was observed in subgroups stratified by age, sex, or TOAST classification ( $\mathrm{P}$ interaction $=0.9537,0.6288$ and 0.8233 respectively).

\section{Discussion}

Significantly increased risk of cognitive impairment was only found in patients with both elevated hsCRP and Hcy levels in our study. The associations among 
Table I Baseline Characteristics of Patients Included versus Not Include

\begin{tabular}{|c|c|c|c|}
\hline Characteristic & All Other Patients & Patients Included & $P$ value \\
\hline No. (\%) & $1159(44.15)$ & $1466(55.85)$ & \\
\hline Age, median (IQR) & $61.00(53.00-68.00)$ & $62.00(54.00-70.00)$ & $0.0493 *$ \\
\hline Female, (\%) & $311(26.83)$ & $4 \mid 4(28.24)$ & 0.4235 \\
\hline BMI, median (IQR) & $24.98(23.32-27.04)$ & $24.69(22.84-26.83)$ & $0.01096 *$ \\
\hline Education, (\%) & & & 0.4755 \\
\hline Illiteracy or primary school & $360(31.06)$ & $438(29.88)$ & \\
\hline Middle School & $422(36.4 I)$ & $518(35.33)$ & \\
\hline High school or higher & $377(32.53)$ & $510(34.79)$ & \\
\hline Current smoker, (\%) & $420(36.24)$ & $52 I(35.54)$ & 0.7107 \\
\hline Heavy drinker, (\%) & $193(16.65)$ & $232(15.83)$ & 0.5679 \\
\hline Hypertension, (\%) & $708(61.09)$ & $934(63.7 I)$ & 0.1678 \\
\hline Diabetes, (\%) & $245(21.14)$ & $354(24.15)$ & 0.0682 \\
\hline Hyperlipidaemia, (\%) & $99(8.54)$ & $160(10.91)$ & $0.043^{*}$ \\
\hline SBP, median, mmHg & $146.00(\mid 33.50-160.00)$ & $147.50(\mid 32.00-162.50)$ & 0.83245 \\
\hline Hcy, median, $\mu \mathrm{mol} / \mathrm{L}$ & $16.30(12.90-22.20)$ & $16.55(13.10-21.90)$ & 0.58202 \\
\hline White blood cell counts, $10^{9} / \mathrm{L}$ & $6.70(5.67-8.11)$ & $6.70(5.64-8.06)$ & 0.5094 \\
\hline hsCRP, mg/L & $1.65(0.76-3.94)$ & $1.50(0.73-3.75)$ & 0.54385 \\
\hline Folate, $\mathrm{nmol} / \mathrm{L}$ & $9.82(5.60-15.30)$ & $9.42(5.22-15.49)$ & 0.6088 \\
\hline Vitamin BI2 (pmol/L); & $259.50(176.00-436.50)$ & $249.00(175.00-378.00)$ & 0.26276 \\
\hline $\mathrm{mRS}$ score before the onset of index events & & & 0.8214 \\
\hline $0-2$ & $1119(96.55)$ & $14 \mid 3(96.38)$ & \\
\hline $3-5$ & $40(3.45)$ & $53(3.62)$ & \\
\hline NIHSS at admission, median (IQR) & $2.00(1.00-4.00)$ & $3.00(1.00-5.00)$ & 0.05659 \\
\hline
\end{tabular}

Note: ${ }^{*} \mathrm{p}<0.05$

Abbreviations: BMI, body mass index; SBP, systolic blood pressure; Hcy, homocysteine; hsCRP, high-sensitivity C reactive protein; mRS, modified Rankin Scale; NIHSS, National Institutes of Health Stroke Scale; IQR, interquartile range.

inflammation, homocysteine, and cognitive impairment were significant in patients who were male, older (over 65 years), and those with the TOAST classification of LAA type. For each MoCA subgroup, no significant interaction for the impact on PSCI was observed in subgroups stratified by age, sex, or TOAST classification.

Previous reports indicated hyperhomocysteinemia as a unique risk factor for cognitive impairment, ${ }^{24,25}$
Sachdev et al in 2003 found that high Hcy level was associated with cognitive impairment, especially in frontal executive functions. In 2019, a prospective multi-center registry conducted by Zhu et al showed that a risk model based on combined tHcy, rheumatoid factor (RF), and matrix metalloproteinase-9 (MMP-9) might enhance the predictive power for cognitive impairment after stroke. ${ }^{26}$ Furthermore, an observational study discovered that homocysteine level was 


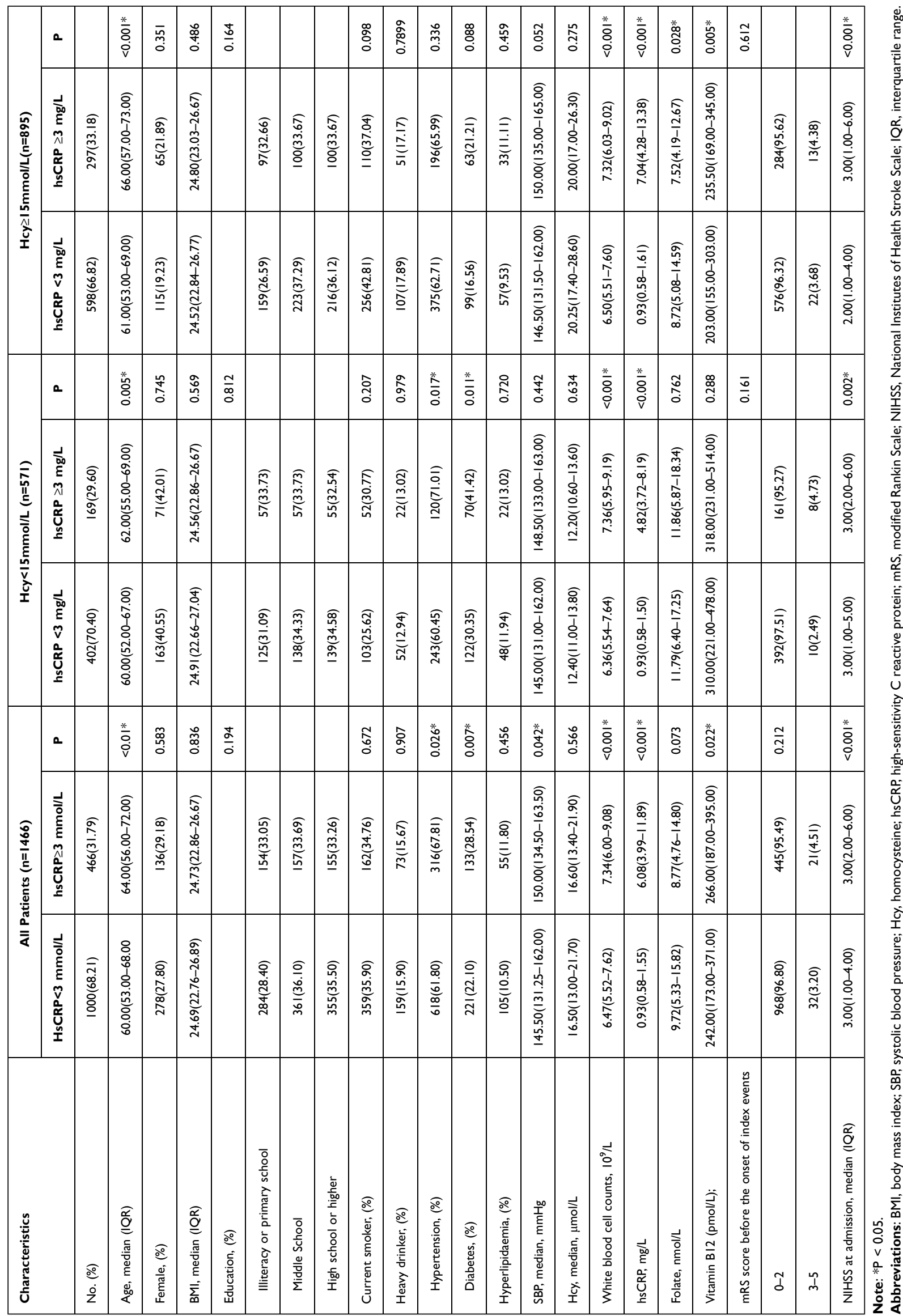


Table 3 Multivariate-Adjusted Odd Ratios (ORs) for Cognitive Impairment According to hsCRP Levels and HCY at 3 Month

\begin{tabular}{|c|c|c|c|c|c|}
\hline & No. (\%) & Crude OR, $95 \% \mathrm{CI}$ & $\mathbf{P}$ & Adjusted OR, $95 \% \mathrm{Cl}$ & $\mathbf{P}$ \\
\hline $\mathrm{hsCRP}<3 \mathrm{mmol} / \mathrm{L}$ & $48 I(48.1)$ & I & & I & \\
\hline $\mathrm{hsCRP} \geq 3 \mathrm{mmol} / \mathrm{L}$ & $274(58.8)$ & $1.54(1.23-1.92) *$ & $<0.001$ & $1.27(0.99-1.62)$ & 0.06 \\
\hline \multicolumn{6}{|l|}{$\mathrm{Hcy}<15 \mathrm{mmol} / \mathrm{L}$} \\
\hline hsCRP $<3 \mathrm{mg} / \mathrm{L}$ & $202(67.79)$ & I & & I & \\
\hline hsCRP $\geq 3 \mathrm{mg} / \mathrm{L}$ & $96(32.21)$ & $1.30(0.91-1.87)$ & 0.15 & $1.05(0.70-1.58)$ & 0.8 \\
\hline \multicolumn{6}{|l|}{$\mathrm{Hcy} \geq 15 \mathrm{mmol} / \mathrm{L}$} \\
\hline hsCRP $<3 \mathrm{mg} / \mathrm{L}$ & $279(46.66)$ & I & & I & \\
\hline hsCRP $\geq 3 \mathrm{mg} / \mathrm{L}$ & I78(59.93) & $1.71(1.29-2.27)^{*}$ & $<0.001$ & $1.42(1.04-1.93)^{*}$ & 0.03 \\
\hline Hcy $<15 \mathrm{mmol} / \mathrm{L}, \mathrm{hsCRP}<3 \mathrm{mg} / \mathrm{L}$ & $202(50.25)$ & I & & I & \\
\hline Hcy $<15 \mathrm{mmol} / \mathrm{L}, \mathrm{hsCRP} \geq 3 \mathrm{mg} / \mathrm{L}$ & $96(56.80)$ & $1.30(0.91-1.87)$ & 0.15 & $1.06(0.72-1.56)$ & 0.78 \\
\hline $\mathrm{Hcy} \geq 15 \mathrm{mmol} / \mathrm{L}, \mathrm{hsCRP}<3 \mathrm{mg} / \mathrm{L}$ & $279(46.66)$ & $0.87(0.67-1.12)$ & 0.26 & $0.87(0.66-1.14)$ & 0.31 \\
\hline $\mathrm{Hcy} \geq 15 \mathrm{mmol} / \mathrm{L}, \mathrm{hsCRP} \geq 3 \mathrm{mg} / \mathrm{L}$ & I78(59.93) & $1.48(1.09-2.01) *$ & 0.01 & $1.23(0.88-1.72)$ & 0.23 \\
\hline
\end{tabular}

Notes: Adjusted for age, sex, NIHSS, hypertension, education, hyperlipidemia, diabetes, white blood cell. $* \mathrm{P}<0.05$.

Abbreviations: $\mathrm{Hcy}$, homocysteine; hsCRP, high-sensitivity $\mathrm{C}$ reactive protein; OR, odds ratios; $\mathrm{Cl}$, confidence interval.

correlated with cerebral microbleeds (CMBs) in cognitively impaired patients. ${ }^{27}$ Moreover, a research conducted in 2013 found that hsCRP combined with Hcy could predict the prognosis of AIS patients among the Chinese mainland populations. ${ }^{28}$

High concentrations of homocysteine can directly exert toxic effects on neurons, which may be caused through amino acid-induced toxicity, reactive oxidative stress (ROS), endoplasmic reticulum, and N-methyl-D-aspartate receptor (NMDAr)-mediated neurotoxicity, leading to neuronal death. ${ }^{29-32}$ In addition, homocysteine may cause vascular endothelial function damage and alter permeability of blood-brain barrier, resulting in cerebral small vessel disease. Combined with the above findings, we speculate that Hcy induces cognitive dysfunction through direct effects on glutamatergic neurotransmission and endothelin, indirect inhibition of methylation processes, enhancement of amyloid neurotoxicity, and promotion of tau phosphorylation. ${ }^{33}$ In addition, previous studies have found that endothelial inflammation under high Hcy conditions promoted vascular injury, ${ }^{34}$ which in turn led to cognitive impairment. However, the underlying mechanisms remain obscure. On the one hand, Hcy can stimulate CRP expression through ROS and mitogen activated protein kinase (MAPK) signaling pathways, ${ }^{35}$ which in turn activates NF- $\kappa \mathrm{B}$ and further promotes inflammation in rat vascular smooth muscle cells. ${ }^{36}$ On the other hand, homocysteine also enhances the expression of NMDAr, ${ }^{35}$ thus stimulating CRP production and triggering inflammatory responses. ${ }^{37}$ Finally, Hcy has been shown to promote neuroinflammation and microglia activation through STAT3 activation subsequent to the ischemic stroke. ${ }^{38}$ Meanwhile, it has been reported that chronic mild hyperhomocysteinemia could cause damage on nuclear acids and protein, as well as ultrastructural alterations in the cerebral cortex in an inflammation-dependent manner. ${ }^{39}$

In subgroup analysis, for patients with both high homocysteine and hsCRP levels, those who were older, male, with a TOAST classification of atherosclerosis type had a higher risk of cognitive impairment compared to those with normal hsCRP levels. Age independently influences MoCA score, ${ }^{3}$ and high age generally reflects a high level of chronic inflammation in the body because CRP level increases with age. ${ }^{40}$ Moreover, female stroke patients seem to have a worse clinical outcome due to distinct hormone levels. ${ }^{41,42}$ In addition, homocysteine is an independent risk factor for atherosclerosis, ${ }^{43}$ which may be attributed to increased collagen synthesis, oxidative damage, endothelial dysfunction, and degradation of elastic material. Meanwhile, CRP is directly involved in the progression of atherosclerosis, a typical chronic inflammatory 




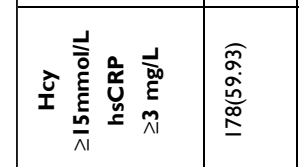

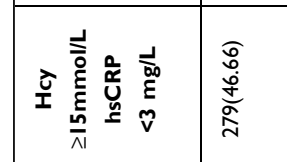

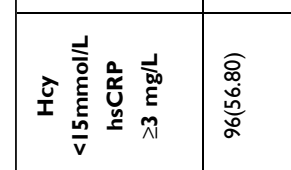

品



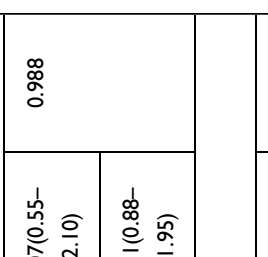

는

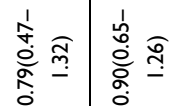

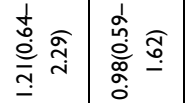

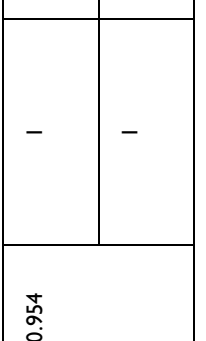

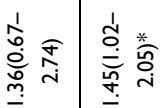

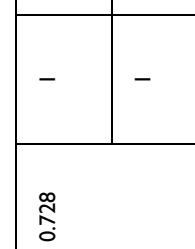

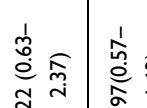

तᄀ

$-$

ริ

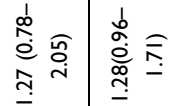

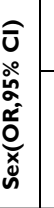

\begin{tabular}{|c|c|}
\hline \multicolumn{2}{|l|}{$\frac{9}{7}$} \\
\hline 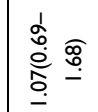 & 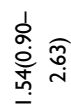 \\
\hline 尊 & 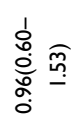 \\
\hline 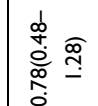 & $\begin{array}{l}\frac{1}{d} \\
\stackrel{2}{0} \\
\stackrel{0}{\infty}\end{array}$ \\
\hline
\end{tabular}

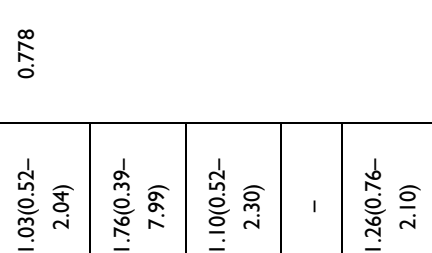

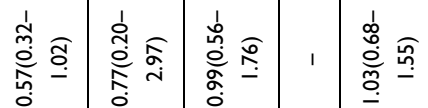

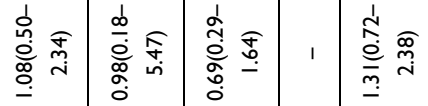

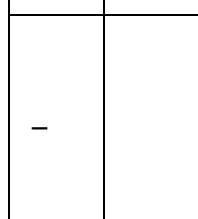

త్రి

$\underset{\infty}{\infty}$

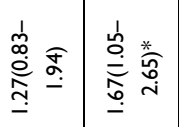

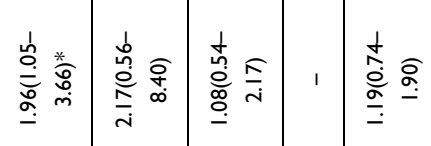

\begin{tabular}{|l|l|}
\hline- & - \\
\hline
\end{tabular}

$\frac{0}{0}$

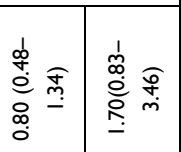

$-$

$\underline{0}$

$\div$

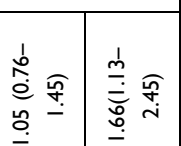

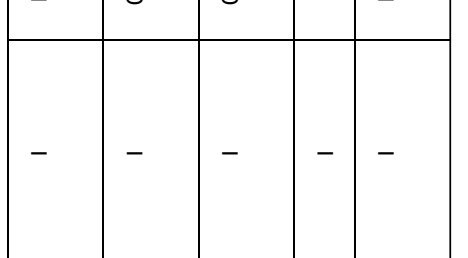

\begin{tabular}{l|l|l|l|l|}
- & - & - & - & - \\
\hline
\end{tabular}

흠

กิ

$\frac{\ddot{0}}{\frac{0}{\underline{\underline{y}}}}$

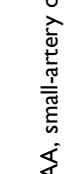

定 
disease, ${ }^{44,45}$ by participating in multiple inflammatory processes. $^{46}$ In light of the above findings, we examined a stratified analysis of age, sex, and TOAST classification in this study, but no interactions were found.

There are several limitations in our study. First, part of the patients in this study experienced a more than 24 $\mathrm{h}$ time period from onset to admission, and thus, their CRP and Hcy levels on admission could not accurately reflect the authentic situation at the time of stroke. Secondly, we only obtained baseline Hcy and CRP levels without any follow-up measures, which made it impossible to study the association between changes in hsCRP and Hcy and cognitive impairment. Thirdly, we stratified the patients into groups according to the hsCRP and Hcy level and make comparisons of clinical characteristics between groups, which will cause the problem of increased type 1 error by multiple comparison. Fourthly, genetic analysis was not included in this study, while MTHFRTT homozygote for homocysteine genotype was confirmed to be related to the increased homocysteine level. ${ }^{27}$ Finally, bias existed when selecting hospitalized patients. Some older patients with other serious diseases may refuse admission at an early stage, and they may have higher levels of both homocysteine and inflammation.

\section{Conclusion}

Our study shows that in patients with hyperhomocysteine post AIS and TIA, high hsCRP concentrations increase the risk of cognitive impairment.

\section{Abbreviations}

Hcy, homocysteine; hsCRP, high sensitive C-reaction protein; AIS, acute ischemic stroke; TIA, transient ischemic attack; PSCI, post-stroke cognitive impairment; ICONS, Impairment of Cognition and Sleep; CNSR-3, China National Stroke Registry-3; MoCA, Montreal Cognitive Assessment; NIHSS: National Institutes of Health Stroke Scale; mRS, modified Rankin Scale; TOAST: Trial of Org 10172 in Acute Stroke Treatment, LAA, large artery atherosclerosis, CE: Cardioembolism; SAA: small-artery occlusion; BMI, body mass index; SBP, systolic blood pressure; $\mathrm{IQR}$, interquartile range; $\mathrm{OR}$, odds ratio; $\mathrm{CI}$, confidence interval.

\section{Data Sharing Statement}

Data are available on reasonable request.

\section{Ethics Approval}

This study was approved by the medical Ethics Committee of Beijing Tiantan Hospital (No.: KY2015-001- 01). All the study participants provided informed consent to take part in this study, in accordance with the Declaration of Helsinki.

\section{Acknowledgments}

We thank all study participants, of the survey teams at participating hospitals of the Third China National Stroke Registry, and the project development and management teams at the Beijing Tiantan Hospital.

\section{Author Contributions}

All authors contributed to data analysis, drafting or revising the article, have agreed on the journal to which the article will be submitted, gave final approval of the version to be published, and agree to be accountable for all aspects of the work.

\section{Funding}

This work was supported by grants from National Key R\&D Program of China (2016YFC0901002), grants from the National Natural Science Foundation of China (81870905, U20A20358).

\section{Disclosure}

The authors report no conflicts of interest in this work.

\section{References}

1. Rothwell PM, Algra A, Amarenco P. Medical treatment in acute and long-term secondary prevention after transient ischaemic attack and ischaemic stroke. Lancet. 2011;377(9778):1681-1692. doi:10.1016/ S0140-6736(11)60516-3

2. Wang YJ, Li Z-X, Gu H-Q, et al. China Stroke Statistics 2019: a Report From the National Center for Healthcare Quality Management in Neurological Diseases, China National Clinical Research Center for Neurological Diseases, the Chinese Stroke Association, National Center for Chronic and Non-communicable Disease Control and Prevention, Chinese Center for Disease Control and Prevention and Institute for Global Neuroscience and Stroke Collaborations. Stroke Vasc Neurol. 2020;5(3):211-239. doi:10.1136/ svn-2020-000457

3. Pendlebury ST, Rothwell PM. Prevalence, incidence, and factors associated with pre-stroke and post-stroke dementia: a systematic review and meta-analysis. Lancet Neurol. 2009;8(11):1006-1018. doi:10.1016/S1474-4422(09)70236-4

4. de Haan RJ, Limburg M, Van der Meulen JHP, et al. Quality of life after stroke. Impact of stroke type and lesion location. Stroke. 1995;26 (3):402-408. doi:10.1161/01.STR.26.3.402

5. Xu Y, Wang Q, Qu Z, et al. Protective effect of hyperbaric oxygen therapy on cognitive function in patients with vascular dementia. Cell Transplant. 2019;28(8):1071-1075. doi:10.1177/0963689719853540 
6. Welch GN, Loscalzo J, Epstein FH. Homocysteine and atherothrombosis. $N$ Engl $J$ Med. 1998;338(15):1042-1050. doi:10.1056/NEJM199804093381507

7. Majumder A, Singh M, George AK, et al. Remote ischemic conditioning as a cytoprotective strategy in vasculopathies during hyperhomocysteinemia: an emerging research perspective. $J$ Cell Biochem. 2019;120(1):77-92. doi:10.1002/jcb.27603

8. Diakoumopoulou E, Tentolouris N, Kirlaki E, et al. Plasma homocysteine levels in patients with type 2 diabetes in a Mediterranean population: relation with nutritional and other factors. Nutr Metab Cardiovasc Dis. 2005;15(2):109-117. doi:10.1016/j.numecd.2004.01.001

9. Cascella M, Arcamone M, Morelli E, et al. Erratum to: multidisciplinary approach and anesthetic management of a surgical cancer patient with methylene tetrahydrofolate reductase deficiency: a case report and review of the literature. J Med Case Rep. 2015;9(1):218. doi:10.1186/s13256-015-0706-5

10. Brustolin S, Giugliani R, Felix TM. Genetics of homocysteine metabolism and associated disorders. Braz J Med Biol Res. 2010;43 (1):1-7. doi:10.1590/S0100-879X2009007500021

11. He Y, Li Y, Chen Y, et al. Homocysteine level and risk of different stroke types: a meta-analysis of prospective observational studies. Nutr Metab Cardiovasc Dis. 2014;24(11):1158-1165. doi:10.1016/j. numecd.2014.05.011

12. Wang C-Y, Chen Z-W, Zhang T, et al. Elevated plasma homocysteine level is associated with ischemic stroke in Chinese hypertensive patients. Eur J Intern Med. 2014;25(6):538-544. doi:10.1016/j.ejim.2014.04.011

13. Levine DA, Wadley VG, Langa KM, et al. Risk factors for poststroke cognitive decline: the REGARDS study (Reasons for Geographic and Racial Differences in Stroke). Stroke. 2018;49(4):987-994. doi:10.1161/STROKEAHA.117.018529

14. Smith AD, Refsum H. Homocysteine, B vitamins, and cognitive impairment. Annu Rev Nutr. 2016;36(1):211-239. doi:10.1146/ annurev-nutr-071715-050947

15. Sanchez-Autet M, Arranz B, Safont G, et al. Gender differences in $\mathrm{C}$-reactive protein and homocysteine modulation of cognitive performance and real-world functioning in bipolar disorder. $J$ Affect Disord. 2018;229:95-104. doi:10.1016/j.jad.2017.12.038

16. Myers GL, Christenson RHM, Cushman M, et al. National Academy of Clinical Biochemistry Laboratory Medicine Practice guidelines: emerging biomarkers for primary prevention of cardiovascular disease. Clin Chem. 2009;55(2):378-384. doi:10.1373/clinchem.2008.115899

17. Yang G, Li C, Wang W, et al. Risk factors for cognitive impairment in patients with first-time ischemic stroke. Am J Transl Res. 2021;13 (3):1884-1889.

18. Zheng F, Xie W. High-sensitivity C-reactive protein and cognitive decline: the English Longitudinal Study of Ageing. Psychol Med. 2018;48(8):1381-1389. doi:10.1017/S0033291717003130

19. Wang Y, Jing J, Meng X, et al. The Third China National Stroke Registry (CNSR-III) for patients with acute ischaemic stroke or transient ischaemic attack: design, rationale and baseline patient characteristics. Stroke Vasc Neurol. 2019;4(3):158-164. doi:10.1136/svn-2019-000242

20. Majumder A, Singh M, George AK, et al. Hydrogen sulfide improves postischemic neoangiogenesis in the hind limb of cystathionine- $\beta$ synthase mutant mice via PPAR- $\gamma /$ VEGF axis. Physiol Rep. 2018;6 (17):e13858. doi:10.14814/phy2.13858

21. Pearson TA, Mensah GA, Alexander RW, et al. Markers of inflammation and cardiovascular disease: application to clinical and public health practice: a statement for healthcare professionals from the Centers for Disease Control and Prevention and the American Heart Association. Circulation. 2003;107(3):499-511. doi:10.1161/01. CIR.0000052939.59093.45

22. Sivakumar L, Kate M, Jeerakathil T, et al. Serial Montreal cognitive assessments demonstrate reversible cognitive impairment in patients with acute transient ischemic attack and minor stroke. Stroke. 2014;45(6):1709-1715. doi:10.1161/STROKEAHA.114.004726
23. Nasreddine ZS, Phillips NA, Bã $\odot$ dirian V, et al. The Montreal cognitive assessment, MoCA: a brief screening tool for mild cognitive impairment. $J$ Am Geriatr Soc. 2005;53(4):695-699. doi:10.1111/j.1532-5415.2005.53221.x

24. Khedr EM, Hamed SA, El-Shereef HK, et al. Cognitive impairment after cerebrovascular stroke: relationship to vascular risk factors.. Neuropsychiatr Dis Treat. 2009;5:103-116. doi:10.2147/ndt.s4184

25. Ji Y, Li X, Teng Z, et al. Homocysteine is associated with the development of cerebral small vessel disease: retrospective analyses from neuroimaging and cognitive outcomes. J Stroke Cerebrovasc Dis. 2020;29(12):105393. doi:10.1016/j.jstrokecerebrovasdis.2020.105393

26. Zhu Z, Zhong C, Guo D, et al. Multiple biomarkers covering several pathways improve predictive ability for cognitive impairment among ischemic stroke patients with elevated blood pressure. Atherosclerosis. 2019;287:30-37. doi:10.1016/j.atherosclerosis.2019.05.028

27. Yoo JS, Ryu C-H, Kim YS, et al. Homocysteinemia is associated with the presence of microbleeds in cognitively impaired patients. J Stroke Cerebrovasc Dis. 2020;29(12):105302. doi:10.1016/j.jstrokecerebro vasdis.2020.105302

28. Tu W-J, Zhao S-J, Liu T-G, et al. Combination of high-sensitivity C-reactive protein and homocysteine predicts the short-term outcomes of Chinese patients with acute ischemic stroke. Neurol Res. 2013;35(9):912-921. doi:10.1179/1743132813Y.0000000228

29. Deep SN, Mitra S, Rajagopal S, et al. GluN2A-NMDA receptor-mediated sustained $\mathrm{Ca}(2+)$ influx leads to homocysteine-induced neuronal cell death. J Biol Chem. 2019;294 (29):11154-11165. doi:10.1074/jbc.RA119.008820

30. Lipton SA, Rosenberg PA. Excitatory amino acids as a final common pathway for neurologic disorders. $N$ Engl J Med. 1994;330 (9):613-622. doi:10.1056/NEJM199403033300907

31. Sibarov DA, Giniatullin R, Antonov SM. High sensitivity of cerebellar neurons to homocysteine is determined by expression of GluN2C and GluN2D subunits of NMDA receptors. Biochem Biophys Res Commun. 2018;506(3):648-652. doi:10.1016/j.bbrc.2018.10.140

32. Majumder A, Singh M, Behera J, et al. Hydrogen sulfide alleviates hyperhomocysteinemia-mediated skeletal muscle atrophy via mitigation of oxidative and endoplasmic reticulum stress injury. $\mathrm{Am}$ J Physiol Cell Physiol. 2018;315(5):C609-C622. doi:10.1152/ ajpcell.00147.2018

33. den Heijer T, Vermeer SE, Clarke R, et al. Homocysteine and brain atrophy on MRI of non-demented elderly. Brain. 2003;126 (1):170-175. doi:10.1093/brain/awg006

34. Huang CF, Wang W-N, Sun -C-C, et al. Echinocystic acid ameliorates hyperhomocysteinemia-induced vascular endothelial cell injury through regulating NF-kappaB and CYP1A1. Exp Ther Med. 2017;14 (5):4174-4180. doi:10.3892/etm.2017.5097

35. Pang X, Liu J, Zhao J, et al. Homocysteine induces the expression of C-reactive protein via NMDAr-ROS-MAPK-NF-kappaB signal pathway in rat vascular smooth muscle cells. Atherosclerosis. 2014;236 (1):73-81. doi:10.1016/j.atherosclerosis.2014.06.021

36. Hidiroglou N, Gilani GS, Long L, et al. The influence of dietary vitamin E, fat, and methionine on blood cholesterol profile, homocysteine levels, and oxidizability of low density lipoprotein in the gerbil. $J$ Nutr Biochem. 2004;15(12):730-740. doi:10.1016/j.jnutbio.2004.04.009

37. Price BR, Wilcock DM, Weekman EM. Hyperhomocysteinemia as a risk factor for vascular contributions to cognitive impairment and dementia. Front Aging Neurosci. 2018;10:350. doi:10.3389/fnagi.2018.00350

38. Chen S, Dong Z, Cheng M, et al. Homocysteine exaggerates microglia activation and neuroinflammation through microglia localized STAT3 overactivation following ischemic stroke. $J$ Neuroinflammation. 2017;14 (1):187. doi:10.1186/s12974-017-0963-x

39. de SMD, Figueiró PW, Siebert C, et al. Chronic mild hyperhomocysteinemia alters inflammatory and oxidative/nitrative status and causes protein/DNA damage, as well as ultrastructural changes in cerebral cortex: is acetylsalicylic acid neuroprotective? Neurotox Res. 2018;33(3):580-592. doi:10.1007/s12640-017-9847-1 
40. Cao C, Hu J, Dong Y, et al. Gender differences in the risk factors for endothelial dysfunction in Chinese hypertensive patients: homocysteine is an independent risk factor in females. PLoS One. 2015;10(2): e0118686. doi:10.1371/journal.pone.0118686

41. Bakeberg MC, Jefferson A, Riley M, et al. Elevated serum homocysteine levels have differential gender-specific associations with motor and cognitive states in parkinson's disease. Parkinsons Dis. 2019;2019:3124295. doi:10.1155/2019/3124295

42. Zhong $\mathrm{C}, \mathrm{Xu} \mathrm{T}, \mathrm{Xu} \mathrm{T}$, et al. Plasma homocysteine and prognosis of acute ischemic stroke: a gender-specific analysis from CATIS randomized clinical trial. Mol Neurobiol. 2017;54(3):2022-2030. doi: 10.1007/s12035-016-9799-0
43. Bautista LE, Arenas IA, Peñuela A, et al. Total plasma homocysteine level and risk of cardiovascular disease: a meta-analysis of prospective cohort studies. J Clin Epidemiol. 2002;55(9):882-887. doi:10.1016/S0895-4356(02)00434-1

44. Libby P, Okamoto Y, Rocha VZ, et al. Inflammation in atherosclerosis: transition from theory to practice. Circ J. 2010;74(2):213-220. doi:10.1253/circj.CJ-09-0706

45. Ross R, Epstein FH. Atherosclerosis - an inflammatory disease. $N$ Engl J Med. 1999;340(2):115-126. doi:10.1056/NEJM199901143400207

46. Devaraj S, Singh U, Jialal I. The evolving role of C-reactive protein in atherothrombosis. Clin Chem. 2009;55(2):229-238. doi:10.1373/ clinchem.2008.108886

\section{Publish your work in this journal}

Neuropsychiatric Disease and Treatment is an international, peerreviewed journal of clinical therapeutics and pharmacology focusing on concise rapid reporting of clinical or pre-clinical studies on a range of neuropsychiatric and neurological disorders. This journal is indexed on PubMed Central, the 'PsycINFO' database and CAS, and is the official journal of The International Neuropsychiatric Association (INA). The manuscript management system is completely online and includes a very quick and fair peer-review system, which is all easy to use. Visit http://www.dovepress.com/testimonials.php to read real quotes from published authors. 